violation and predicts relationships between $\mathrm{CP}$ effects. Our major aim is to find out if these relationships work or not."

Present estimates of $\mathrm{CP}$ violation are based on observations of another subatomic particle, the $\mathrm{K}$ meson or kaon, but far better measurements should be possible from studying decay channels of B mesons. Pursuit of that goal led to fierce competition between the Cornell storage ring and SLAC to build a B-factory, and to the even more ambitious Japanese project.

The time it will take to obtain results depends on what they are. If the measurement of CP violation falls in the range expected from the study of kaons, a more accurate measurement should be made quickly. If the result is smaller, it will take longer.

"This could be a hundred-yard dash, or it could be a marathon," says Jonathan Dorfan, the South African physicist who oversaw the B-factory's construction and replaced Richter as SLAC's director last September. "By the end of the year, we should have enough data to confront the issue of CP violation."

Although the BaBar detector is comparable in scale to others built in the United States, it has unusually strong non-US participation. The United States is paying $\$ 68$ million of the detector's $\$ 110$ million cost, with the rest coming from abroad, mainly from Europe. SLAC has attracted similar international participation in another major project, the Gamma-ray Large Area Space Telescope, which it hopes to build for the US space agency NASA (see box).

Dorfan admits that high-energy physicists in the United States need results from the Bfactory to demonstrate that they are still making progress on big scientific questions. "The field needs some discoveries," he says.

After obtaining an initial estimate of CP violation, both $\mathrm{B}$-factories are expected to study rarer B-meson decay channels (some of which occur only a few times every million events) to improve understanding of $\mathrm{B}$

\title{
Sky's the limit as teams bid for NASA project
}

\section{Stanford, California}

Physicists at the Stanford Linear Accelerator (SLAC) are hoping to secure a vital new line of business for their laboratory next week, when the US space agency NASA is expected to announce plans for a satellite to succeed the defunct Compton Gamma Ray Observatory.

NASA will choose between rival proposals from SLAC and from its own Marshall Space Flight Centre at Huntsville, Alabama, and Washington University, St Louis, to build the main instrument for the Gammaray Large Area Space Telescope
(GLAST), due to be launched in 2005. "We're expecting that we'll win," says Jonathan Dorfan, SLAC's director.

SLAC's confidence is partly based on the enthusiasm professed by Dan Goldin, the administrator of NASA, for using detector technologies developed by high-energy physicists in space. Under SLAC's proposal, NASA, the Department of Energy and a group of overseas collaborators would each carry a third of the $\$ 120$ million cost of a GLAST instrument that would use thin silicon solid-state detectors to track gamma-ray bursts. NASA would pay an additional $\$ 200$ million to launch the spacecraft.

The project would "bring the culture of high-energy physics to work with NASA on an instrument designed and built by scientists", says David Leith, former head of the research division at SLAC. The detector would be a hundred times as sensitive as the EGRET instrument carried by the Compton observatory. The rival proposal from the Marshall Space Flight Center would use fibreoptics technology to detect the gamma-ray signals. mesons and perhaps help theorists amend the Standard Model. To this end, SLAC plans upgrades that could increase the luminosity of its B-factory by an order of magnitude over five years. It hopes to make substantial progress before 2008, when a B-meson experiment is due to begin at the Large Hadron Collider at CERN, the Genevabased European Laboratory for Particle Physics, generating a tidal wave of data on the decay of trillions of B mesons. Colin Macilwain

Robert Triendl adds from Tokyo: Japan's Bfactory project, Belle, has reached about one-fifteenth of its projected luminosity output. Scientists at Belle say that the SLAC B-factory has achieved 1.7 to 1.8 times the luminosity of the Japanese machine, though physicists at SLAC say they are doing better than that (see above).

Several possible explanations for Belle's limited performance are being investigated, says a spokesperson for the facility, including interactions between the positron beam and clouds of photoelectrons generated by synchrotron radiation, which may result in the beam being unfocused and blurred.

The Stanford group made efforts to deal with this problem at an early stage of the design, but observers say the Japanese design group may have underestimated the effect. Scientists at Belle say it is not yet clear why the beam is unfocused. "We are investigating various possibilities," says one. "We still think a technical breakthrough to optimize the performance of the accelerator is possible."

Several strategies to refocus the beam and optimize performance are being considered, including minimizing the photoelectron effect by replacing beam pipes and installing magnets to trap photoelectrons.

A spokesperson for Belle claims that the luminosity problems have had little effect on the scheduling of experiments. The main detectors and related data-acquisition systems are almost completed. "We hope to be able to provide some clues to $\mathrm{CP}$ violation by this summer," he says.

\section{Former director made enemies but put SLAC on the road to success.}

\section{Department of Energy laboratories.}

But Martha Krebs, assistant secretary for science at the department until late last year, praised Richter's ability to "tell it the way it really is" on behalf of all the lab directors. That role "has not been reproduced" by the other directors since Richter quit, she said.

Richter's frankness and acerbic sense of humour have made him plenty of enemies, however. Guests at the celebration included the directors of CERN (the European Laboratory for Particle Physics) and Japan's KEK, but present and past directors of Fermilab, for example, did not attend.

Some high-energy physicists think that
Richter's lack of support behind the scenes badly injured the Superconducting Super Collider project at the time of its 1993 collapse. In an interview, Richter said of the episode: "I faced a very difficult problem. As the SSC's costs went up, there was a group who said it was the SSC or nothing they said, if that meant shutting SLAC, then shut it." It was the SSC, of course, which was closed.

Richter looks now to a new generation of leaders - Jonathan Dorfan at SLAC and Michael Witherall at Fermilab, and their counterparts abroad — to put past disagreements behind them.
"The good thing is that the new directors can blame the old ones for the problems that exist," he says.

While returning to research at SLAC, Richter is currently serving as president of the International Union of Pure and Applied Physics. He now looks to the Internet to help suspend the turf wars that adorned the second half of his career.

"We've got to get over this business of price and place," he told the physicists. "We are the inventors of the World-Wide Web, but we haven't taken advantage of it to make it irrelevant where an accelerator is built." 